\title{
Covf-981056 -
}

\section{On Routing Algorithms With End-To-End Delay Guarantees;}

\author{
Nageswara S. V. Rao \\ Oak Ridge National Laboratory \\ Oak Ridge, Tennessee 37831-6355 \\ Stephen G. Batsell \\ Oak Ridge National Laboratory \\ Oak Ridge, Tennessee 37831-6367 \\ sgb@ornl.gov \\ "The submitted manuscript has been \\ authored by a contractor of the U.S. \\ Government under contract No. DE- \\ AC05-96OR22464. Accordingly, the \\ U.S. Government retains a nonexclu- \\ sive, royalty-free license to publish or \\ sive, royalty free license to publish or \\ reproduce the published form of thi \\ contribution, or allow others to do
for U.S. Government purposes."
}

Computer Science and Mathematics Division

Computing, Information, and Networking Division

Submitted to Seventh International Conference on Computer Communications and Networks, October 12-15, 1998, Lafayette, Louisiana.

\section{IASTER HPT $^{T}$}

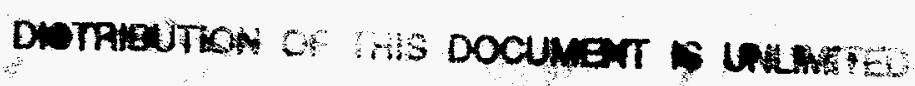

$\lceil$ Research sponsored by the Laboratory Directed Research and Development Program of Oak Ridge National Laboratory, managed by Lockheed Martin Energy Research Corp. for the U. S. Department of Energy under Contract No. DE-AC05-96OR22464. 


\section{DISCLAIMER}

This report was prepared as an account of work sponsored by an agency of the United States Government. Neither the United States Government nor any agency thereof, nor any of their employees, makes any warranty, express or implied, or assumes any legal liability or responsibility for the accuracy, completeness, or usefulness of any information, apparatus, product, or process disclosed, or represents that its use would not infringe privately owned rights. Reference herein to any specific commercial product, process, or service by trade name, trademark, manufacturer, or otherwise does not necessarily constitute or imply its endorsement, recommendation, or favoring by the United States Government or any agency thereof. The views and opinions of authors expressed herein do not necessarily state or reflect those of the United States Government or any agency thereof. 


\section{DISCLAIMER}

Portions of this document may be illegible in electronic image products. Images are produced from the best available original document. 


\title{
On Routing Algorithms With End-to-End Delay Guarantees ${ }^{1}$
}

\author{
Nageswara S. V. Rao \\ Computer Science and Mathematics Division \\ Oak Ridge National Laboratory \\ Oak Ridge, Tennessee 37831-6355 \\ Stephen G. Batsell \\ Computing, Information, and Networking Division \\ Oak Ridge National Laboratory \\ Oak Ridge, Tennessee 37831-6367 \\ sgb@ornl.gov
}

\begin{abstract}
We consider the transmission of a message of size $r$ from a source to a destination with guarantees on the end-to-end delay over a computer network with $n$ nodes and $m$ links. There are three sources of delays: (a) propagation delays along the links, (b) delays due to bandwidth availability on the links, and (c) queuing delays at the intermediate nodes. First, we consider that delays on various links and nodes are given as functions of the message size. If the delay in (b) is a non-increasing function of the bandwidth, we propose $O\left(m^{2}+m n \log n\right)$ time algorithm to compute a path with the minimum end-to-end delay for any given message size $r$. We then consider that the queuing delay in (c) is a random variable correlated with the message size according to an unknown distribution. At each node, the measurements of queuing delays and message sizes are available. We propose two algorithms to compute paths whose delays are close to optimal delays with a high probability, irrespective of the distribution of the delays, and based entirely on the measurements of sufficient size.
\end{abstract}

Keywords and Phrases: End-to-end delay, quality of service, routing, probably approximately correct, path-table.

\section{Introduction}

End-to-end delay guarantees are very important in computer networks that are expected to support a variety of services requiring transmission of messages of varying sizes. In the present networks, especially the Internet, such guarantees cannot be provided, and a message can indeed be arbitrarily delayed under certain load conditions. In the future, however, newer applications such as medical image transfer and real-time instrumentation control over networks, require that messages be sent with certain guarantees on the end-to-end delay. Since the requirements can be quite varied, different "dedicated" network architectures might be required for various applications. The exact nature of the guarantees that can be provided over such networks depends on the delay sources and their precise contribution to the end-to-end delay.

In this paper, we propose polynomial-time source-based algorithms for computing a path with end-to-end delay guarantees under fairly general delay profiles, which are specified either as (deterministic) functions or as measurements of random delay variables. In the first case, we present an exact algorithm to compute a path with the minimum end-to-end delay for any given message

\footnotetext{
${ }^{1}$ Research sponsored by the Laboratory Directed Research and Development Program of Oak Ridge National Laboratory, managed by Lockheed Martin Energy Research Corp. for the U. S. Department of Energy under Contract No. DE-AC05-96OR22464.
} 
size. In the second case, we consider that the transmission is subject to random queuing delays with unknown distributions. Based on the measurements of queuing delays, we propose two algorithms that yield paths which approximate the optimal paths (computable only under a complete knowledge of delay distributions) with a high probability, irrespective of delay distributions. The closeness of approximation depends on the sample size and the size of the network.

We consider a computer network represented by a graph $G=(V, E)$ with $n$ nodes and $m$ edges or links. A message of size $r$ must be transmitted from a source node $s$ to a destination node $d$ with performance guarantees on the end-to-end delay. In a deterministic formulation, a message transmitted on the network incurs three types of delays:

(a) Link Propagation Delay: For each link $e=\left(v_{1}, v_{2}\right)$, there is a link-delay $d(e) \geq 0$ such that a message of unit length sent via $e$ from node $v_{1}$ at time $t$ will arrive at node $v_{2}$ at time $t+d(e)$. The link-delay essentially includes the propagation time of the link.

(b) Bandwidth Constrained Delay: Each link $e \in E$ has a bandwidth $b(e) \geq 0$. Once initiated, a message of $r$ units can be sent along link $e$ in $g(r, b(e))+d(e)$ time, where $g(r, b)$ is non-decreasing in $r$ and non-increasing in $b$. For a simple bandwidth constraint, we have $g(r, b(e))=r / b(e)[5]$ so that the delay along link $e$ is $r / b(e)+d(e)$.

(c) Deterministic Queuing Delay: At any node $v$, there is a deterministic queuing delay $q_{v}(r)$ which is a function of the message size $r$.

Consider a simple path $P$, from source $s=v_{0}$ to destination $d=v_{k}$, given by $\left(v_{0}, v_{1}\right),\left(v_{1}, v_{2}\right)$, $\ldots,\left(v_{k-1}, v_{k}\right)$, where $\left(v_{j}, v_{j+1}\right) \in E$, for $j=0,1, \ldots,(k-1)$, and $v_{0}, v_{1}, \ldots, v_{k}$ are distinct. Subsequently, a simple path is referred to simply as a path. Let $e_{j}=\left(v_{j}, v_{j+1}\right)$. The bandwidth of this path, denoted by $b(P)$, is given by $\underset{j=0}{k-1} b\left(e_{j}\right)$, and the delay due to bandwidth is given by $g(r, b(P))$. Then, the end-to-end delay of path $P$ in transmitting a message of size $r$ is given by

$$
t(r, P)=g(r, b(P))+\sum_{j=0}^{k-1} d\left(e_{j}\right)+\sum_{j=0}^{k-1} q_{j}(r) .
$$

Let us denote $d(P)=\sum_{j=0}^{k-1} d\left(e_{j}\right)$ and $q(r, P)=\sum_{j=0}^{k-1} q_{v_{j}}(r)$ such that $t(r, P)=g(r, b(P))+d(P)+$ $q(r, P)$. We show that a path with the minimum end-to-end delay for any given message size $r$ can be computed with time complexity $O\left(m^{2}+m n \log n\right)$. A special case of this problem, when $q(r, P)=0$ and $g(r, b(P))=r / b(P)$, for all $P$, has been extensively studied under the appellation of the quickest path problem $[5,14,9]$.

The algorithm for the above deterministic formulation is applicable to special networks wherein delay functions are accurately known. In more general networks, however, good estimates may be available only for link delays and bandwidths, but the queuing delays are only known approximately at best. Typically, the queuing delays depend on the messages from other sources competing to be sent on the same link. In practice, however, empirical measurements of the queuing delays can be collected at each node by logging the delays and sizes of all messages routed through it. To address the problems of this type, we consider a probabilistic formulation of the problem with the queuing delays characterized as follows: 
$\left(c^{\prime}\right)$ A message of size $R$ arrives at the source $s$ according to an unknown distribution $P_{R}{ }^{2}$. At any node $v, Q_{v}$ and $R_{v}$ are the random variables ${ }^{3}$ denoting the queuing delay and message size distributed according to unknown distributions $P_{Q_{v}}$ and $P_{R_{v}}$, respectively. Note that $Q_{v}$ is not a simple function of $R_{v}$ in that for any given value of $R_{v}$ there could be more than one value for $Q_{v}$. The measurements $\left(Q_{v ; 1}, R_{v ; 1}\right),\left(Q_{v ; 2}, R_{v ; 2}\right), \ldots,\left(Q_{v ; l}, R_{v ; l}\right)$ that are independently and identically distributed (iid) according to the joint distribution $P_{Q_{v}, R_{v}}$, are known at each node $v \in V$. For any given message size $R_{v}=r$, let $\bar{q}_{v}(r)$ denote the expectation of $Q_{v}$, i. e. $\bar{q}_{v}(r)=E\left[Q_{v} \mid R_{v}=r\right]$. For the second algorithm, we assume that $\bar{q}_{v}(r)$ is a non-decreasing function of $r$ which informally means that longer messages are likely to be more delayed than shorter ones ${ }^{4}$. Note that this condition does not imply that the values of $Q_{v ; i}$ plotted against $R_{v ; i}$ are non-decreasing.

We consider two routing problems under the probabilistic formulation for which the algorithm developed under the conditions (a)-(c) can be utilized. The end-to-end delay of path $P$ in transmitting a message of size $R$ is

$$
T(R, P)=g(R, b(P))+d(P)+\sum_{j=0}^{k-1} Q_{v_{j}}
$$

which is a random variable of the joint distributions of queuing delays at various nodes and the message size. The expected delay of path $P$ averaged over all message sizes is given by

$$
\bar{T}(P)=\int g(R, b(P)) d P_{R}+d(P)+\sum_{j=0}^{k-1} \int Q_{v_{j}} d P_{Q_{v_{j}}, R_{v_{j}}} .
$$

Let $P^{*}$ denote a path with the minimum expected end-to-end delay, i.e. $\bar{T}\left(P^{*}\right)=\min _{P \in \mathcal{P}} \bar{T}(P)$, where $\mathcal{P}$ is set of all paths between $s$ and $d$. We present an algorithm to compute a path $\hat{P}$ based entirely on iid measurements, such that given a sample of size

$$
l=\frac{32(n+1)^{2} \tau^{2}}{\epsilon^{2}}\left[4 \ln (4(n+1) \tau / \epsilon)+\ln \left(2^{n} 18(n+1) / \delta\right)\right]
$$

we have

$$
P\left[\left|\bar{T}(\hat{P})-\bar{T}\left(P^{*}\right)\right| \geq \epsilon\right] \leq \delta
$$

where $\sup _{r, b} g(r, b) \leq \tau_{1}, \sup _{v} Q_{v} \leq \tau_{2}$, and $\tau=\max \left(\tau_{1}, \tau_{2}\right)$. This performance guarantee means that with a high probability the expected delay of the computed path is within $\epsilon$ of the optimal expected delay irrespective of the underlying distributions.

We consider the end-to-end delay of a path $P$ for a given message size $R$ given by

$$
T_{R}(P)=g(R, b(P))+d(P)+\sum_{j=0}^{k-1} Q_{v_{j} \mid R}
$$

\footnotetext{
${ }^{2}$ We denote the random variables by capital letters (e. g. $R$ ) and their deterministic counterparts by the corresponding lower case letter (e. g. r).

${ }^{3}$ For the existence of $Q_{v}$ the sizes of the external messages arriving at all source nodes (not just $s$ ) that are routed via $v$ be probabilistic (in particular, the existence of $P_{R}$ at $s$ alone is not sufficient). Furthermore, it is assumed that for any given $R$ the routing path is deterministically chosen for all sources in the network.

${ }^{4}$ This is not true for certain queuing disciplines such as the first-in and first-out.
} 
The expected delay of path $P$ for a given message size $R$ is given by

$$
\bar{T}_{R}(P)=g(R, b(P))+d(P)+\sum_{j=0}^{k-1} \int Q_{v_{j}} d P_{Q_{v_{j}} \mid R} .
$$

Let $P_{R}^{*}$ be the path with minimum end-to-end delay for message size $R$, i.e. $\bar{T}_{R}\left(P_{R}^{*}\right)=\min _{P \in \mathcal{P}} \bar{T}_{R}(P)$. By using regression estimation, we present an algorithm to compute $\hat{P}_{R}$ such that

$$
P\left[\left|T_{R}\left(\hat{P}_{R}\right)-T_{R}\left(P_{R}^{*}\right)\right| \geq \epsilon\right] \leq \delta
$$

for a sufficiently large sample. Informally, this condition guarantees that the delay of $\hat{P}_{R}$ is close to that of $P_{R}^{*}$ with a high probability. Performance guarantees of the type in Eq (1.3) and (1.4) are similar in spirit to those in Probably Approximately Correct (PAC) learning $[15,16]$. Such results are about the best one can expect in scenarios where very little information is available about the underlying probability distributions.

The present problem is a special case of the well-known optimal routing problem [3], and can also be posed as a special case of the classical minimum cost flow problem [1] studied in transportation and operations research. The main contributions of this paper are polynomial-time algorithms, and PAC guarantees based only on measurements, i.e. without the knowledge of distributions.

A number of Quality of Service (QoS) routing algorithms that provide bounds on end-to-end delay and/or transmission rates have been studied based on single paths $[18,17]$ and multiple paths $[10,12]$. Optimization of network-level parameters is discussed in [10], and their QoS parameters are not deterministic nor distribution-free. In spirit, our problem is a special case of the one studied in [7], where the transmission task is specified by several parameters, but our algorithms are polynomial-time computable and source-based. More general variations of the problem are discussed in $[2,8]$, but their guarantees are "soft" and are not measurement-based.

The organization of this paper is as follows. An algorithm for computing the minimum endto-end delay path under the conditions $(a)$ - $(c)$ is presented in Section 2. The problem of random queuing delays under the conditions $(a),(b)$, and $\left(c^{\prime}\right)$ is addressed in Section 3.

\section{Minimum End-To-End Delay Routing}

We now describe an algorithm to compute a path with the minimum end-to-end delay according to Eq. (1.1) for transmitting a message of given size $r$ from $s$ to $d$. We define an augmented delay of an edge $e=\left(v_{1}, v_{2}\right)$ as $d_{A}(e)=d(e)+q_{v_{1}}(r)$. Let $b_{1}, b_{2}, \ldots, b_{c}$ denote the distinct values of the bandwidths $b(e), e \in E$. Let $G(a)=(V, E(a))$ denote the subnetwork where $e \in E(a)$ if and only if $b(e) \geq a$. Let a $s-d$ shortest path in $G(a)$ denote the shortest delay path based only on the augmented delay of the edges (i.e. with minimum $d_{A}($.$) value). Our algorithm Min-Path is based$ on the algorithm of [14] which was originally proposed for the special case when $q_{v}(r)=0$, and $g(r, b(P))=r / b(P)$.

Step 1 of this algorithm is executed by $c$ invocations of Dijkstra's shortest path algorithm with a total complexity $O(c m+c n \log n)$ using Fibonacci heaps [6]. The cost of step 2 is $O(c)$. Thus the complexity of this algorithm is $O\left(m^{2}+m n \log n\right)$. We establish the correctness of this algorithm in the following theorem, which is an extension of the results from [14]. 
Theorem 2.1 Let $P^{t}$ be a path with the minimum end-to-end delay in $G$ for message size $r$, i. e. $t\left(P^{t}\right)=\min _{P \in \mathcal{P}} t(P)$. Then the following statements are true:

(i) $P^{t}$ is a $s-d$ shortest path in $G\left(b\left(P^{t}\right)\right)$ according to the augmented delay $d_{A}($.$) .$

(ii) Let $P_{j}$ be a $s-d$ shortest path in $G\left(b_{j}\right), j=1,2, \ldots, c$, and

$$
g\left(r, b\left(P_{l}\right)\right)+d\left(P_{l}\right)+q\left(r, P_{l}\right)=\min _{j=1}^{c}\left\{g\left(r, b\left(P_{j}\right)\right)+d\left(P_{j}\right)+q\left(r, P_{j}\right)\right\}
$$

Then $P_{l}$ is a minimum end-to-end delay path in $G$ for the message size $r$.

Proof: For Part (i), consider any path $P_{1}$ in $G\left(b\left(P^{t}\right)\right)$ which implies $b\left(P_{1}\right) \geq b\left(P^{t}\right)$. This condition in turn implies $q\left(r, b\left(P_{1}\right)\right) \leq q\left(r, b\left(P^{t}\right)\right)$. Since $P^{t}$ is a minimum end-to-end delay path, we have

$$
q\left(r, b\left(P^{t}\right)\right)+d\left(P^{t}\right)+q\left(r, P^{t}\right) \leq q\left(r, b\left(P_{1}\right)\right)+d\left(P_{1}\right)+q\left(r, P_{1}\right),
$$

which implies $d\left(P^{t}\right)+q\left(P^{t}, r\right) \leq d\left(P_{1}\right)+q\left(P_{1}, r\right)$. Thus Part (i) is true.

We now consider Part (ii). For the minimum end-to-end delay path $P^{t}$, we have $b\left(P^{t}\right)=b_{j_{0}} \in$ $\left\{b_{1}, b_{2}, \ldots, b_{c}\right\}$. By Part (i), $P^{t}$ is shortest in $G\left(b_{j_{0}}\right)$ and $P_{j_{0}}$ is shortest in $G\left(b_{j_{0}}\right)$ by hypothesis. Thus we have $d\left(P^{t}\right)+q\left(r, P^{t}\right)=d\left(P_{j_{0}}\right)+q\left(r, P_{j_{0}}\right)$. Furthermore, we have $b\left(P_{j_{0}}\right) \geq b\left(P^{t}\right)$ since all edges of $G\left(b_{j_{0}}\right)$ have the bandwidth of at least $b_{j_{0}}$. Thus, we have $g\left(r, b\left(P_{j_{0}}\right)\right) \leq g\left(r, b\left(P^{t}\right)\right)$, which implies $g\left(r, b\left(P_{j_{0}}\right)\right)+d\left(P_{j_{0}}\right)+q\left(r, P_{j_{0}}\right) \leq g\left(r, b\left(P^{t}\right)\right)+d\left(P^{t}\right)+q\left(r, P^{t}\right)$. By the minimization hypothesis of the theorem we have $g\left(r, b\left(P_{l}\right)\right)+d\left(P_{l}\right)+q\left(r, P_{l}\right) \leq g\left(r, b\left(P_{j_{0}}\right)\right)+d\left(P_{j_{0}}\right)+q\left(r, P_{j_{0}}\right)$. In summary, we have $t\left(r, P_{l}\right) \leq t\left(r, P_{j_{0}}\right) \leq t\left(r, P^{t}\right)$.

Although the algorithm appears to be applicable only to the cases when a complete knowledge of the delays is available, it could be useful when only estimators of the delays are available.

\section{Routing Based on Measurements}

The expected end-to-end delay of path $P$ is given by

$$
\bar{T}(P)=\int g(R, b(P)) d P_{R}+d(P)+\sum_{j=0}^{k-1} \int Q_{v_{j}} d P_{Q_{v_{j}}, R_{v_{j}}} .
$$

Since the distributions $P_{R}$ and $P_{Q}=P_{Q_{v_{1}}, \ldots Q_{v_{n}}}$ are unknown, the best expected end-to-end delay path, $\bar{P}$, such that $\bar{T}(\bar{P})=\min _{P \in \mathcal{P}} \bar{T}(P)$, cannot be computed. In this section, we first present a method to compute $\hat{P}$ that guarantees the condition in Eq (1.3). The expected end-to-end delay for a given message size $R$ can be written as follows

$$
\bar{T}_{R}(P)=g(R, b(P))+d(P)+\sum_{j=0}^{k-1} \bar{q}_{v_{j}}(R)
$$

where $\bar{q}_{v}(r)=E\left[Q_{v} \mid R=r\right]$ is the regression of $Q_{v}$ on $R$. Under the additional condition that $\bar{q}_{v}(r)$ is non-decreasing in $r$, we employ a regression estimator $\hat{q}_{v}($.$) to compute a path \hat{P}_{R}$ such that $T_{R}\left(\hat{T}_{R}\right)$ is close to $T_{R}\left(P_{R}^{*}\right)$ as in $\mathrm{Eq}(1.4)$.

algorithm Min-Path $(r)$

1. for $j=1,2, \ldots, c$, compute $s-d$ shortest path $P_{j}$ in $G\left(b_{j}\right)$;

2. compute index $k$ which minimizes $\left\{g\left(r, b\left(P_{j}\right)\right)+d\left(P_{j}\right)+q\left(r, P_{j}\right) \mid j=1,2, \ldots, c\right\}$;

3. return $P_{k}$ as the path with the minimum end-to-end delay; 


\subsection{Best Empirical Path Method}

We consider the empirical end-to-end delay given by

$$
\hat{T}(P)=\frac{1}{l} \sum_{i=1}^{l} g\left(R_{i}, b(P)\right)+\sum_{j=0}^{k-1} d\left(e_{j}\right)+\sum_{j=0}^{k-1} \frac{1}{l} \sum_{i=1}^{l} Q_{v_{j} ; i} .
$$

Note that $\hat{T}($.$) can be computed since it involves only the measured quantities. Then the best$ empirical end-to-end delay path is defined by $\hat{P}=\arg \underset{P \in \mathcal{P}}{\min } \hat{T}(P)$, which is computed using the algorithm described in Section 2 . Note that $\hat{P}$ is best on the average based on measurements alone.

Theorem 3.1 Under the conditions (a)-(c), given a sample of size

$$
l=\frac{32(n+1)^{2} \tau^{2}}{\epsilon^{2}}\left[4 \ln (4(n+1) \tau / \epsilon)+\ln \left(2^{n} 18(n+1) / \delta\right)\right],
$$

the best empirical path $\hat{P}$ can be computed in $O\left(m^{2}+m n \log n\right)$ time such that

$$
P\left[\left|\bar{T}(\hat{P})-\bar{T}\left(P^{*}\right)\right| \geq \epsilon\right] \leq \delta,
$$

where $\sup _{r, b} g(r, b) \leq \tau_{1}, \sup _{v} Q_{v} \leq \tau_{2}$, and $\tau=\max \left(\tau_{1}, \tau_{2}\right)$.

Proof: Consider that we have $P\left[\sup _{P \in \mathcal{P}}|\bar{T}(P)-\hat{T}(P)| \geq \epsilon / 2\right]<\delta$

for some $\delta>0$. Then, with probability at least $1-\delta$, we have $|\bar{T}(P)-\hat{T}(P)| \leq \epsilon / 2$ for all paths simultaneously. By noting that $\hat{T}(\hat{P}) \leq \hat{T}(\bar{P})$, we have

$$
\bar{T}(\hat{P}) \leq \hat{T}(\hat{P})+\epsilon / 2 \leq \hat{T}(\bar{P})+\epsilon / 2 \leq \bar{T}(\bar{P})+\epsilon
$$

with probability $1-\delta$. Thus we have $P\left[\left|\bar{T}(\hat{P})-\bar{T}\left(P^{*}\right)\right| \geq \epsilon\right] \leq P\left[\sup _{P \in \mathcal{P}}|\bar{T}(P)-\hat{T}(P)| \geq \epsilon / 2\right]$.

We now bound the right hand side as follows

$$
\begin{aligned}
P\left[\sup _{P \in \mathcal{P}}|\bar{T}(P)-\hat{T}(P)| \geq \epsilon\right] \leq & P\left[\sup _{P \in \mathcal{P}}|\bar{g}(b(P))-\hat{g}(b(P))| \geq \frac{\epsilon}{2(n+1)}\right] \\
& +P\left[\sup _{P \in \mathcal{P}}\left|\sum_{j=0}^{k-1} \bar{Q}_{v_{j}}-\sum_{j=0}^{k-1} \hat{Q}_{v_{j}}\right| \geq \frac{\epsilon n}{2(n+1)}\right],
\end{aligned}
$$

where we have $\bar{g}(b(P))=\int g(R, b(P)) d P_{R} \quad$ and $\quad \hat{g}(b(P))=\sum_{i=1}^{l} g\left(b(P), R_{i}\right)$,

$$
\bar{Q}_{v}=\int Q_{v} d P_{Q_{v}, R_{v}} \quad \text { and } \quad \hat{Q_{v}}=\frac{1}{l} \sum_{i=1}^{l} Q_{v ; i}
$$

for $v \in V .{ }^{5}$ The first term of Eq. (3.1) is upper bounded as follows

$$
\begin{aligned}
P\left[\sup _{P \in \mathcal{P}}|\bar{g}(b(P))-\hat{g}(b(P))| \geq \frac{\epsilon}{2(n+1)}\right] & \leq P\left[\sup _{i=1,2, \ldots, m}\left|\bar{g}\left(b_{i}\right)-\hat{g}\left(b_{i}\right)\right| \geq \frac{\epsilon n}{2(n+1)}\right] \\
& \leq m 18 l e^{-\frac{\epsilon^{2} \ell}{16(n+1)^{2} r^{2}}},
\end{aligned}
$$

\footnotetext{
${ }^{5}$ Here we applied the basic result that $P\left[A+B>\epsilon_{1}+\epsilon_{2}\right] \leq P\left[A>\epsilon_{1}\right]+P\left[A>\epsilon_{2}\right]$ since the event $A+B>\epsilon_{1}+\epsilon_{2}$ implies that event that either $A>\epsilon_{1}$ or $A>\epsilon_{2}$ (or both); we apply this basic result a number of times in the proofs of this section.
} 
where the last step follows from by Glivenka-Cantelli Lemma ([16], p. 184)). The second term of Eq. (3.1) is upper bounded as follows

$$
\begin{aligned}
P\left[\sup _{P \in \mathcal{P}}\left|\sum_{j=0}^{k-1} \bar{Q}_{v_{j}}-\sum_{j=0}^{k-1} \hat{Q}_{v_{j}}\right| \geq \frac{\epsilon n}{2(n+1)}\right] & \leq 2^{n} \sup _{P \in \mathcal{P}} P\left[\left|\sum_{j=0}^{k-1} \bar{Q}_{v_{j}}-\sum_{j=0}^{k-1} \hat{Q}_{v_{j}}\right| \geq \frac{\epsilon n}{2(n+1)}\right] \\
& \leq 2^{n} \sup _{P \in \mathcal{P}} \sup _{v \in P} P\left[\left|\bar{Q}_{v}-\hat{Q}_{v}\right| \geq \frac{\epsilon}{2(n+1)}\right] \\
& \leq 2^{n} n 18 l \epsilon^{\frac{-\epsilon^{2} l}{16(n+1)^{2} \tau^{2}}},
\end{aligned}
$$

where the first inequality is due to $|\mathcal{P}| \leq 2^{n}$, the second inequality is because there are at most $n$ nodes in any $P$, and the third inequality is due to Glivenko-Cantelli Lemma. In summary, we have

$$
P\left[\sup _{P \in \mathcal{P}}|\bar{T}(P)-\hat{T}(P)| \geq \epsilon / 2\right] \leq 18\left(m+2^{n} n\right) l e^{-\frac{\epsilon^{2} l}{16(n+1)^{2} \tau^{2}}} \leq 2^{n} 18(n+1) l e^{-\frac{\epsilon^{2} l}{16(n+1)^{2} \tau^{2}}}
$$

The sample bound follows by noting that the condition $\delta=a l e^{-b l}$ can be ensured by choosing $l \geq \frac{2}{b} \ln \left(\frac{a}{b^{2} \delta}\right)$.

Notice that the sample size is proportional to $O\left(n^{3}\right)$ for fixed values of parameters $\epsilon$ and $\delta$, with no knowledge about the underlying delay distributions. In statistics literature, asymptotic convergence results are more common. Under the condition of Theorem 3.1, we can show that $\bar{T}(\hat{P}) \rightarrow \bar{T}\left(P^{*}\right)$ with probability one by a direct application of Borel-Cantelli Lemma [4].

\subsection{Regression Method}

The path $\hat{P}$ of previous section does not fully exploit the given value of message size. We now present a method that utilizes the correlation information between message size and queuing delay by utilizing an estimator $\hat{q}_{v}($.$) of the regression \bar{q}_{v}($.$) . Intuitively, this method "extrapolates" the$ measurements to the present value of $R$, and computes the corresponding best path. Consider, for a given message size $R$,

$$
\hat{T}_{R}(P)=g(R, b(P))+d(P)+\sum_{j=0}^{k-1} \hat{q}_{v_{j}}(R) .
$$

Let $\hat{P}_{R}$ minimize $\hat{T}_{R}($.$) , i.e. \hat{T}\left(\hat{P}_{R}\right)=\min _{P \in \mathcal{P}} \hat{T}_{R}(P)$, which can be computed by the algorithm of Section 2. Let $\mathcal{Q}$ denote set of all non-decreasing functions, and we consider a particular estimator $\hat{q}_{v}(.) \in \mathcal{Q}$ that minimizes the empirical cost function given by $\frac{1}{l} \sum_{q \in \mathcal{Q}}\left|Q_{v ; i}-f\left(R_{v ; i}\right)\right|$ for $f \in \mathcal{Q}$. We emphasize that for computing $\hat{P}_{R}$ it not necessary to have any measurements at the message size $R$. Thus, a main contribution of the regression method is the computation of paths with performance guarantees at message sizes for which measurements may not be available.

Theorem 3.2 Consider that the regression $\bar{q}_{v}(r)=E\left[Q_{v} \mid R_{v}=r\right]$ is non-decreasing in $r$. Under the conditions $(a),(b)$ and $\left(c^{\prime}\right)$, given a sample of size

$$
l=\frac{32 n^{2} \tau_{2}^{2}}{\epsilon^{2}}\left[4 \ln \left(4 n \tau_{2} / \epsilon\right)+\ln \left(2^{n} 72 n / \delta\right)\right]
$$

the path $\hat{P}_{R}$ can be computed in $O\left(m^{2}+m n \log n\right)$ time such that $P\left[\left|T_{R}\left(\hat{P}_{R}\right)-T_{R}\left(P_{R}^{*}\right)\right| \geq \epsilon\right] \leq \delta$, where $\sup _{v} Q_{v} \leq \tau_{2}$. 
Proof: We have

$$
\begin{aligned}
P\left[\left|T_{R}\left(\hat{P}_{R}\right)-T_{R}\left(P_{R}^{*}\right)\right|>\epsilon\right] \leq & P\left[\left|T_{R}\left(\hat{P}_{R}\right)-\bar{T}_{R}\left(\hat{P}_{R}\right)\right|>\epsilon_{1}\right] \\
& +P\left[\left|\bar{T}_{R}\left(\hat{P}_{R}\right)-\bar{T}_{R}\left(P_{R}^{*}\right)\right|>\epsilon-\epsilon_{1}-\epsilon_{2}\right] \\
& +P\left[\left|\bar{T}_{R}\left(P_{R}^{*}\right)-T_{R}\left(P_{R}^{*}\right)\right|>\epsilon_{2}\right] .
\end{aligned}
$$

By Chebyshev's inequality, we have for any $\epsilon_{1}, \epsilon_{2}>0$,

$$
\begin{aligned}
& P\left[\left|T_{R}\left(\hat{P}_{R}\right)-\bar{T}_{R}\left(\hat{P}_{R}\right)\right|>\epsilon_{1}\right] \leq \frac{1}{\epsilon_{1}} E\left[\left|T_{R}\left(\hat{P}_{R}\right)-\bar{T}_{R}\left(\hat{P}_{R}\right)\right|\right] \\
& P\left[\left|\bar{T}_{R}\left(P_{R}^{*}\right)-T_{R}\left(P_{R}^{*}\right)\right|>\epsilon_{2}\right] \leq \frac{1}{\epsilon_{2}} E\left[\left|\bar{T}_{R}\left(P_{R}^{*}\right)-T_{R}\left(P_{R}^{*}\right)\right|\right]
\end{aligned}
$$

Since $E\left|T_{R}\left(\hat{P}_{R}\right)-\bar{T}_{R}\left(\hat{P}_{R}\right)\right|=0$, and $E\left|\bar{T}_{R}\left(P_{R}^{*}\right)-T_{R}\left(P_{R}^{*}\right)\right|=0$, we have

$$
P\left[\left|T_{R}\left(\hat{P}_{R}\right)-T_{R}\left(P_{R}^{*}\right)\right|>\epsilon\right] \leq P\left[\left|\bar{T}_{R}\left(\hat{P}_{R}\right)-\bar{T}_{R}\left(P_{R}^{*}\right)\right|>\epsilon\right] .
$$

We now upper bound the right hand side. First, we have $\bar{T}_{R}\left(\hat{P}_{R}\right)-\bar{T}_{R}\left(P_{R}^{*}\right) \leq \sum_{j=0}^{k-1}\left|\bar{q}_{v_{j}}(r)-\hat{q}_{v_{j}}(r)\right|$. By utilizing the result of Vapnik [16] (as in the first part of proof of Theorem 3.1), we have

$$
\begin{aligned}
P\left[\left|\bar{T}_{R}\left(\hat{P}_{R}\right)-\bar{T}_{R}\left(P_{R}^{*}\right)\right|>\epsilon\right] & \leq P\left[\sup _{P \in \mathcal{P}}\left|\bar{T}_{R}(P)-\hat{T}_{R}(P)\right|>\epsilon / 2\right] \\
& \leq 2^{n} \sup _{P \in \mathcal{P}} P\left[\left|\bar{T}_{R}(P)-\hat{T}_{R}(P)\right|>\epsilon / 2\right] \\
& \leq 2^{n} n \sup _{P \in \mathcal{P}} \sup _{v \in P} P\left[\left|\bar{q}_{v}(R)-\hat{q}_{v}(R)\right|>\epsilon / 2 n\right] .
\end{aligned}
$$

We note that Vapnik's capacity of $\mathcal{Q}$ is 2 which yields the following bound for any $v \in V$ (see [16] for details)

$$
P\left[\left|\bar{q}_{v}(R)-\hat{q}_{v}(R)\right|>\epsilon / 2 n\right] \leq 18 l^{2} e^{-\frac{\epsilon^{2} l}{16 \tau^{2} n^{2}}} .
$$

Thus we have $P\left[\left|\bar{T}_{R}\left(\hat{P}_{R}\right)-\bar{T}_{R}\left(P_{R}^{*}\right)\right|>\epsilon\right] \leq 2^{n} n 18 l^{2} e^{-\frac{\epsilon^{2} l}{1 \epsilon \tau^{2} n^{2}}}$, which yields the required sample size by noting that the condition $\delta=a l^{2} e^{-b l}$ can be ensured by choosing $l \geq \frac{2}{b} \ln \left(\frac{4 a}{b^{2} \delta}\right)$.

Using Borel-Cantelli Lemma it can be shown that $\left|T_{R}\left(\hat{P}_{R}\right)-T_{R}\left(P_{R}^{*}\right)\right| \rightarrow 0$ with probability one. The main objective of this theorem is to demonstrate that regression estimators can be used to provide performance guarantees based on finite samples. Several other regression estimators can be used in place of $\hat{q}$. But, to obtain the required sample size, finite sample results are needed for regression estimation; such results are fairly uncommon since most regression results are asymptotic (with some exceptions $[13,11]$ ). For example, using Nadaraya-Watson estimator $\hat{q}_{v}^{N W}$, sample sizes are estimated in [13] to ensure the condition $P\left[\sup _{r}\left|\bar{q}_{v}(r)-\hat{q}_{v}^{N W}(r)\right|>\epsilon\right] \leq \delta$ under additional smoothness conditions on regressions and densities.

\section{Conclusions}

We consider the transmission of a message of size $r$ from a source to a destination with guarantees on the end-to-end delay over a computer network with $n$ nodes and $m$ links. Under fairly general 
conditions, we proposed polynomial-time algorithms to compute paths whose delays are close to optimal expected delays with a high probability, irrespective of the distribution of delays. These algorithms are obtained by developing efficient algorithms for a general deterministic case, which could be of independent interest. Our main motivation is to establish that QoS guarantees in PAC framework can be provided under fairly general conditions without the knowledge of distributions. Several direct improvements are possible: the sample size estimates can be improved, and more sophisticated regression estimation methods can be employed. The main limitation of the proposed algorithms is the requirement of "stationarity" of the distributions. Extensions of our algorithms to non-stationary cases can be addressed under certain martingale or mixing conditions.

\section{References}

[1] R. K. Ahuja, T. L. Magnati, and J. B. Orlin. Network Flows. Prentice Hall, Englewood Cliffs, NJ, 1993.

[2] C. Aurrecocechea, A. T. Campbell, and L. Hauw. A survey of QoS architectures. Multimedia Systems Journal, 1996.

[3] D. Bertsekas and R. Gallager. Data Networks. Prentice-Hall, 1992.

[4] P. Billingsley. Probability and Measure. John Wiley and Sons, New York, second edition, 1986.

[5] Y. L. Chen and Y. H. Chin. The quickest path problem. Computers and Operations Research, 17(2):153$161,1990$.

[6] T. H. Cormen, C. E. Leiserson, and R. L. Rivest. Introduction to Algorithms. McGraw-Hill Book Co., New York, 1990.

[7] D. Ferrari and D. C. Verma. A scheme for real-time channel establishment in wide-area networks. IEEE Journal on Selected Areas in Communications, 8(3):368-379, 1990.

[8] H. Kanakia, P. P. Mishra, and A. R. Reibman. An adaptive congestion control scheme for real time packet video transport. IEEE/ACM Transactions on Networking, 3(6):671-682, 1995.

[9] E. Q. V. Martins and J. L. E. Santos. An algorithms for the quickest path problem. Operations Research Letters, 20:195-198, 1997.

[10] S. Murthy and J. J. Garcia-Luna-Aceves. Congestion-oriented shortest multipath routing. In Proc. of IEEE INFOCOM'96, 1996.

[11] H. Qiao, N. S. V. Rao, and V. A. Protopopescu. PAC learning of using Nadaraya-Watson estimator based on orthogonal systems. In M. Li and A. Maruoka, editors, Algorithmic Learning Theory. Lecture Notes in Artificial Intelligence 1316, Springer-Verlag, 1997.

[12] N. S. V. Rao and S. G. Batsell. QoS routing via multiple paths using bandwidth reservation. In IEEE INFOCOM98: The Conference on Computer Communications. 1998.

[13] N. S. V. Rao and V. Protopopescu. On PAC learning of functions with smoothness properties using feedforward sigmoidal networks. Proceedings of the IEEE, 84(10):1562-1569, 1996.

[14] J. B. Rosen, S. Z. Sun, and G. L. Xue. Algorithms for the quickest path problem and the enumeration of quickest paths. Computers and Operations Research, 18(6):579-584, 1991.

[15] L. G. Valiant. A theory of the learnable. Communications of the ACM, 27(11):1134-1142, 1984.

[16] V. Vapnik. Estimation of Dependences Based on Empirical Data. Springer-Verlag, New York, 1982.

[17] R. Vogel, R. G. Herrtwich, W. Kalfa, H. Wittig, and L. C. Wolf. QoS-based routing of multimedia streams in computer networks. IEEE Journal on Selected Areas in Communications, 14(7):1235-1244, 1996.

[18] Z. Wang and J. Crowcroft. QOS routing for supporting resource reservation. IEEE Journal on Selected Areas in Communications, 14(7):1228-1234, 1996. 